\section{KURZ GEMELDET}

Allergy News

\section{Augen auf bei alten Antihistaminika}

— reiverkäufliche H1-Antihistaminika der ersten Generation werden nach häufig eingesetzt. Sie wurden jedoch vor dem Zeitalter randomisierter kontrollierter Studien und moderner evidenzbasierter Medizin eingeführt. In einer Metaanalyse setzten sich jetzt Forscher des Global Allergy and Asthma European Network (GA2LEN) mit den Konsequenzen eines willkürlichen Einsatzes ohne ärztliche Aufsicht auseinander. Sie sichteten dazu die Literatur sowie Medienberichte zu Unfällen aus den Jahren 1996 bis 2008, die sich unter einer solchen Medikation ereignet hatten. Das Ergebnis: Die Präparate reduzieren die REM-Schlafphase und setzen das Lernvermögen und die Arbeitsleistung herab. Manche wirken in größeren Mengen kardiotoxisch. Zudem wur-

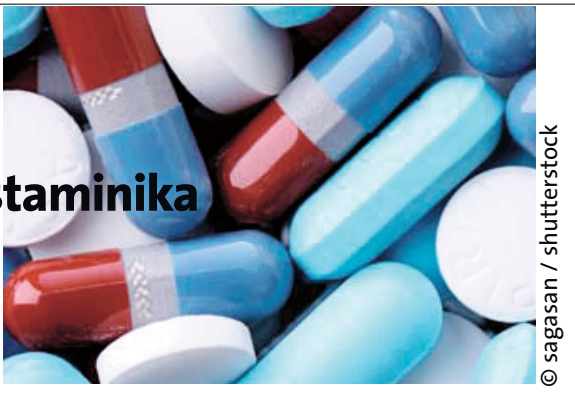

den sie bei Verkehrsunfällen mit Flugzeug, Auto oder Boot sowie bei Todesfällen aufgrund versehentlicher oder absichtlicher Überdosierung nachgewiesen. Die Autoren fordern daher einen besseren Verbraucherschutz und raten, H1-Antihistaminika der ersten Generation nicht länger rezeptfrei zur Verfügung zu stellen. Sie verweisen auf die neueren, nicht sedierenden H1-Antihistaminika der zweiten Generation, die ein besseres Nutzen-RisikoVerhältnis hätten und zu ähnlichen Preisen verfügbar seien.

bsk

Church MK et al. Allergy 2010 Feb 8

[Epub ahead of print]

\title{
Seelische Spätfolgen
}

_ ine atopische Dermatitis in den ers- ten beiden Lebensjahren hinterlässt Spuren in der Kinderseele. Selbst wenn die Ekzeme danach verschwunden sind, haben diese Kinder im Alter von zehn Jahren häufiger psychische Auffälligkeiten, vor allem emotionale Probleme, als Kinder ohne Neurodermitisanamnese. Noch häufiger sind psychische Probleme bei Kindern, deren atopische Dermatitis über das zweite Lebensjahr hinaus bestehen bleibt, so das Ergebnis einer deutschen Studie mit knapp 6.000 Kindern.

Schmitt J et al. J Allerg Clin Immunol

2010;125: 404-10

\section{Exotische Dermatitis}

\section{Der Smiley-Test: Erkennen uns Bienen?}

_ ranzösische Biologen versuchten Bie-

nen darauf zu konditionieren, gesichtsähnliche Strukturen zu erkennen. Tatsächlich flogen die Tiere nach einiger Zeit bevorzugt zu einem einfachen StrichPunkt-Gesicht, bei dem es Zuckerwasser gab, und ließen andere Muster links liegen. Auch ein anschließender Versuch mit Fotos realer Gesichter war erfolgreich. Wurde dagegen bei den Fotos die Position von Augen, Nase oder Mund verändert, behandelten die Tiere die Bilder wie unbekannte Muster. Die Bienen orientieren sich also an der relativen Anordnung der Gesichtsmerkmale. Individuelle Menschen können die Tiere damit allerdings nicht unterscheiden.

bsk

Avarguès-Weber $\mathbf{A}$ et al. J Exp Biol

2010;213: 593-601

\section{Silikon-Allergie bei Herzschrittmacher}

W elche Probleme allergische Reaktionen gegen Silikon bereiten können, zeigt der Fall eines zwölfjährigen Mädchens mit einem Herzschrittmacher. Im Bereich der Schrittmachertasche bildete sich eine Fistel, aus der eine gelatineartige Masse austrat. Infektionserreger waren nicht nachweisbar. Der histologische Befund war vereinbar mit einer Fremd-

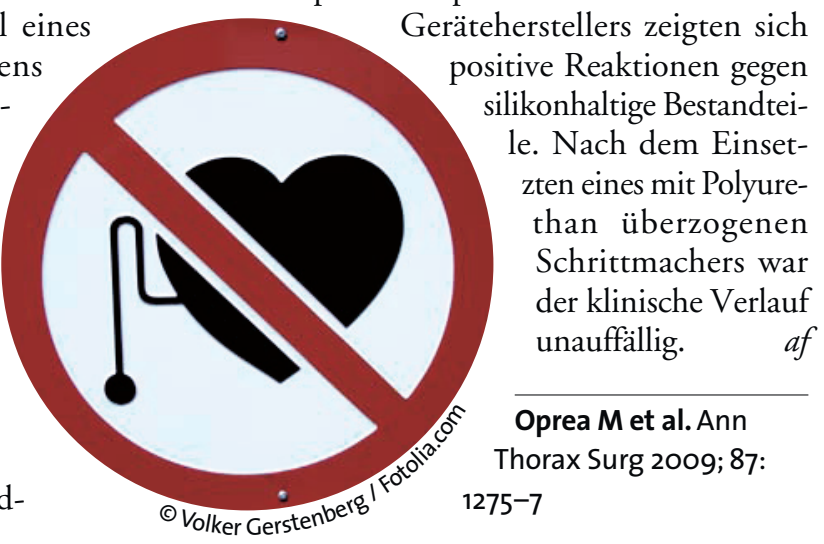

körperreaktion, doch erst in einem komponentenspezifischen Patch-Test des Geräteherstellers zeigten sich onen gegen e. Nach dem Einsetten eines mit PolyureSchrittmachers war der klinische Verlauf unauffällig. af

nn es zur Verschlimmerung und bakteriellen Superinfektion kommen. abd

Litvinov IV et al. Pediatrics 2010;125: e419-22 